\title{
Optical biopsy in gastroenterology: Focus on confocal laser endomicroscopy
}

\author{
Zaheer $\mathrm{Nabi}^{1}$ (D) D. Nageshwar Reddy ${ }^{1}$ \\ Published online: 2 October 2019 \\ (C) Indian Society of Gastroenterology 2019
}

\section{Introduction}

The quality of endoscopy images has improved remarkably over the last several decades. Current generation endoscopes provide high-definition images with superior resolution and are capable of magnification as well. This means that subtle lesions are less likely to be missed on routine endoscopy. However, high definition alone may not be superior to standard white light endoscopy for in vivo characterization of polyps [1]. Differentiation of the lesions as neoplastic vs. non-neoplastic is essential as it bears implication on subsequent management and surveillance strategies. For example, hyperplastic polyps bear no malignant potential. On the other hand, adenomatous polyps harbor a definite risk of malignant transformation and need to be resected. The integration of image enhancement techniques like narrow band imaging (NBI) in the endoscopes have largely overcome the issue of discriminating neoplastic and non-neoplastic polyps in the gastrointestinal (GI) tract. Novel image enhancement techniques allow better characterization of the lesions as compared with high-definition endoscopy alone [2].

\section{Image enhanced and optical endoscopic techniques}

Conventional white light endoscopy is largely inaccurate (accuracy $59 \%$ to $84 \%$ ) in differentiating neoplastic from nonneoplastic polyps. The incorporation of optical techniques in the currently available endoscopes has largely overcome the unmet need generated by standard or high-definition white light endoscopy. Current endoscopic image enhancement techniques

D. Nageshwar Reddy

aigindia@yahoo.co.in

1 Asian institute of Gastroenterology, 6-3-661 Somajiguda, Hyderabad 500 082, India include NBI ( Olympus Medical Co., Tokyo, Japan), i-scan (Pentax Hoya, Tokyo, Japan), flexible spectral imaging color enhancement (FICE, Fujifilm Medical Co., Tokyo, Japan), and blue laser imaging (BLI, Fujifilm Medical Co., Tokyo, Japan). Other techniques in various phases of development include autofluorescence imaging (AFI, Olympus Medical Co, Tokyo, Japan), endocytoscopy, confocal laser endomicroscopy (CLE, Cellvizio System Mauna Kea, Paris, France), optical coherence tomography, multiphoton microscopy, and Raman spectroscopy.

In this issue of the Journal, one such technique, i.e. probebased CLE (pCLE), was evaluated for the characterization of GI lesions in the upper and lower GI tracts [3]. In the following section, we will discuss the current status and the future directions with respect to the applications of CLE in digestive tract.

\section{Confocal laser endomicroscopy: principle and technique}

In CLE, a low-power laser is used to illuminate the tissue of interest. The fluorescence of the light subsequently deflected from the tissue is refocused by the same lens onto the confocal detection system. The presence of a pinhole ensures that only the light reflected from a specific plane gets detected and processed further, whereas the light deflected from other planes and not in line with the pinhole is rejected (Fig. 1). This characteristic feature of CLE enables high spatial resolution and evaluation of tissue architecture at cellular level [4].

There are two basic systems of CLE: endoscope-integrated system (eCLE) and probe-based system (pCLE). Dedicated endoscopes (EC3870K, Pentax, Tokyo, Japan) have integrated CLE system at the tip of the endoscope. On the other hand, pCLE system (Cellvizio Endomicroscopy System; Mauna Kea Technologies, Paris, France) utilizes miniature probes (0.92.5-mm diameter), which can be passed through the accessary channel of most of the commercially available endoscopes. These probes are essentially composed of a fiberoptic bundle 
Confocal system for laser

illumination and detection



Fig. 1 Principle of confocal laser endomicroscopy

with integrated distal lens and can be used approximately 20 times [4]. Various types of confocal miniprobes are available for use in the GI tract including GastroFlex ultra high definition (UHD) (upper GI tract), ColoFlex UHD (colon), CholangioFlex (ERCP), and AQ-Flex 19 (needle-based CLE).

The advantages of eCLE include provision of a wider field of view and an adjustable depth of scanning $(0-250 \mu \mathrm{m})$ as compared with pCLE, in which the depth of scanning $(\sim$ $50 \mu \mathrm{m}$ ) is fixed depending on the type of probe being used. On the other hand, image acquisition rate is faster with pCLE (12 frames/second) as compared with eCLE (0.8-1.6 frames/ second). eCLE system is no longer available for commercial use, and therefore, most studies have evaluated the pCLE system in the GI and hepatobiliary tract.

\section{Contrast agents for CLE}

CLE requires the administration of topical (acriflavine hydrochloride and cresyl violet) and/or intravenous (fluorescein sodium and indocyanine green) fluorescent contrast agents immediately before the procedure. Acriflavine hydrochloride strongly labels the nuclei and superficial epithelial cells, whereas fluorescein sodium does not stain nuclei but enables deeper imaging by highlighting the vasculature, the lamina propria, and the intercellular spaces [5]. Besides the less favorable staining characteristics, there are concerns over the mutagenic potential of acriflavine. Therefore, intravenous fluorescein is generally preferred over the topical contrast agents for CLE.

\section{Technique of pCLE}

In pCLE, the confocal miniprobe is passed through the working channel of the standard endoscope and placed gently in contact with the tissue of interest after the administration of the contrast agent $(2.5-5.0 \mathrm{~mL}$ of $10 \%$ fluorescein sodium). The probe should be perpendicular to the tissue for better image acquisition. Since blood and mucus can interfere with the quality of imaging, the area of interest should be washed and care should be taken while placing the probe on friable tissues. For the same reason, biopsies should be avoided before CLE examination. Optimal images can be obtained from $30 \mathrm{~s}$ to $8-10 \mathrm{~min}$ (up to $60 \mathrm{~min}$ ) after the administration of contrast agents. The images can also be saved and stored for subsequent offline analysis and interpretation.

\section{Current and potential applications of CLE in the GI tract}

In the GI tract, CLE has been evaluated for the following indications: identification of dysplasia in Barrett's esophagus [6], classification of gastric and colorectal polyps (Table 1), assessment of disease activity and development of dysplasia in inflammatory bowel disease [18, 19], evaluation of indeterminate biliary strictures [20], and characterization of solid and cystic pancreatic masses [21, 22]. In addition, limited data suggests that CLE may be potentially useful for the in vivo identification of celiac disease (villous atrophy and increased intraepithelial lymphocytes) [23], ampullary lesions [24], and follow up after endoscopic mucosal resection (EMR) of colorectal polyps [25].

The subsequent discussion will focus on the application and utility of CLE for polypoidal lesions in the GI tract.

\section{CLE for polypoidal lesions in the GI tract}

CLE provides real-time histology of the polyps in both the upper and lower GI tracts. It enables the differentiation of hyperplastic or non-neoplastic polyps from neoplastic polyps with a reasonable degree of accuracy. There are two main classification systems, which are utilized to characterize the dysplastic and non-dysplastic GI epithelium, i.e. Mainz and Miami classification systems.

\section{CLE for gastric polyps}

CLE has been found useful and accurate in characterizing gastric lesions like intestinal metaplasia, gastric intraepithelial neoplasia, adenomas, and cancers. However, there is no well-defined classification system for gastric polyps or lesions, and experts have utilized the cellular morphology and the architecture of glands and vessels to differentiate between different gastric lesions [10]. In the normal gastric mucosa, the glands and epithelial cells are regularly arranged, homogenous with good polarity, and the 
Table 1 Outcomes of confocal laser endomicroscopy in the gastrointestinal tract (gastric and colonic)

\begin{tabular}{|c|c|c|c|c|c|}
\hline Study & $\begin{array}{l}\text { Number of } \\
\text { lesions (number of patients) }\end{array}$ & GI tract & Size (mean or median) & $\begin{array}{l}\text { Sensitivity/ } \\
\text { specificity }\end{array}$ & Accuracy \\
\hline Hurlstone et al. $(2008)^{*}[7]$ & $162(39)$ & Colon & $1-34 \mathrm{~mm}$ & $97.4 \% / 99.3 \%$ & $99.1 \%$ \\
\hline Gomez et al. (2010) [8] & $75(53)$ & Colonic & NA & $76 \% / 72 \%$ & $75 \%$ \\
\hline De Palma et al. (2010) [9] & $32(20)$ & Colorectal & $13 \mathrm{~mm}$ & $100 \% / 84.6 \%$ & $92.3 \%$ \\
\hline \multirow[t]{2}{*}{ Li et al. (2011) [10] } & 182 (phase I) & Gastric lesions & $21 \mathrm{~mm}$ & $84 \% / 92.1 \%$ & $88.5 \%$ \\
\hline & 1786 (phase II) & & $14 \mathrm{~mm}$ & $88.9 \% / 99.3 \%$ & $98.8 \%$ \\
\hline Jeon et al. (2011) [11] & $35(31)$ & Gastric lesions & NA & NA & $94.2 \%$ \\
\hline Shahid et al. (2012) [12] & 130 polyps $(65)$ & Small colorectal polyps & $4.6 \mathrm{~mm}$ & $86 \% / 78 \%$ & $82 \%$ \\
\hline Kuiper et al. (2012) [13] & $154(64)$ & Colorectal lesions & $5.0 \mathrm{~mm}$ & $59.5 \% / 77.4 \%$ & $71.9 \%$ \\
\hline Buchner et al. (2010) [14] & $119(75)$ & Colonic polyps & $10 \mathrm{~mm}$ & $91 \% / 76 \%$ & $91 \%$ \\
\hline Gong et al. (2015) [15] & $86(82)$ & Gastric lesions & $16.7 \mathrm{~mm}$ & $91.9 \% / 90 \%$ & $93.5 \%$ \\
\hline Belderbos et al. (2017) [16] & $113(52)$ & Colonic polyps & $8 \mathrm{~mm}$ & $88 \% / \mathrm{NA}$ & $76 \%$ \\
\hline Chen et al. (2018) [17] & 322 & Gastric lesions & NA & $72.4 \% / 93.1 \%$ & $88.9 \%$ \\
\hline Current study [3] & $50(50)$ & $\begin{array}{l}\text { Upper and lower GI } \\
\text { polypoidal lesions }\end{array}$ & $13.7 \mathrm{~mm}$ & $87.5 \% / 79.1 \%$ & \\
\hline
\end{tabular}

*Confocal laser endoscope

GI gastrointestinal, NA not available

vessels are normal in caliber (honeycomb-like in the gastric body or coil-shaped in the antrum). The presence of villous architecture and goblets cells defines intestinal metaplasia. In contrast, the glandular and cellular polarity are impaired or lost in intraepithelial neoplasia and gastric cancer, respectively. In addition, the vessels are dilated and irregular in caliber in neoplasia.

Several studies have assessed the utility of CLE in the realtime evaluation of gastric pre-neoplastic and neoplastic lesions. In a large study including 182 (phase I) and 1786 (phase II) patients, real-time eCLE had a higher sensitivity ( $88.9 \%$ vs. $72.2 \%$ ), specificity (99.3\% vs. $95.1 \%$ ), and accuracy (98.8\% vs. $94.1 \%$ ) for gastric superficial cancer than white light endoscopy [10]. In another study, CLE was compared with magnifying endoscopy NBI (ME-NBI) in 82 patients with suspected superficial gastric cancers. CLE was highly accurate, but not superior to ME-NBI for differentiating cancerous from noncancerous gastric lesions (accuracy 93.5\% vs. 93.7\%) [15]. A recent systematic review and meta-analysis including twentythree studies analyzed the diagnostic value of CLE for gastric cancer and precancerous lesions among Asian population [26]. The pooled sensitivity and specificity of CLE were $91 \%$ and $99 \%$ in gastric cancer and $81 \%$ and $98 \%$ in gastric intraepithelial neoplasia, respectively [26]. In one study, CLE (accuracy 94.2\%) outperformed endoscopic biopsy (accuracy $85.7 \%$ ) for the diagnosis of gastric adenomas and adenocarcinomas [11]. Therefore, CLE has the potential to replace endoscopic biopsy or at least provide a guidance to obtain targeted biopsy specimens. Unlike colonic polyps, the data is more limited on the application of CLE in gastric lesions. In addition, a standardized classification system is warranted for uniform reporting of CLE images in different gastric lesions.

\section{CLE for colonic polyps}

CLE has been more widely assessed for its role in the evaluation of colonic polyps as compared with gastric polyps. In the initial studies by Kiesslich et al. (2004) and Hurlstone et al. (2008), CLE was highly accurate (99\%) in the prediction of neoplastic changes in the colonic polyps [5, 7]. Of note, integrated confocal endoscope (no longer available) was used in both these studies as compared with majority of the later studies in which the pCLE system was used. Such a high degree of accuracy could not be replicated in the subsequent studies, which revealed a diagnostic yield ranging from $72 \%$ to $99 \%$ (Table 1). Several studies have compared pCLE with the currently established narrow spectrum imaging techniques or virtual chromoendoscopy. Buchner et al. compared pCLE with virtual chromoendoscopy (NBI or FICE) in 75 patients with 119 colonic polyps. The sensitivity of pCLE was better than virtual chromoendoscopy for the classification of polyps (91\% vs. 77\%) [14]. In contrast, the post-hoc accuracy of pCLE was found to be inferior to NBI and chromoendoscopy in a study by Kuiper and colleagues [13]. Poor quality of videos was presumably responsible for the inferior results with pCLE in this study. Similar concerns have been raised in the other studies regarding the acquisition of good-quality pCLE images for offline evaluation [16, 27]. Another factor that may influence the accuracy of in vivo histology is the experience of the operator and inter-observer agreement. There is limited literature regarding the learning curve of pCLE $[28,29]$. In a study by Buchner et al., the accuracy improved from $63 \%$ for the first twenty lesions to $86 \%$ after 60 lesions [28]. A few other studies also concluded that accurate post-hoc interpretation of 
eCLE confocal images can be learned quickly [29, 30]. In addition, the inter-observer agreement for the classification of neoplasia has been found to be moderate to good in another recent study [8]. These studies suggest that interpretation and acquisition of pCLE images are not difficult to learn by novices.

\section{Comparison of outcomes: current study vs. published literature}

In the current issue of the Journal, Goenka and colleagues prospectively evaluated the histology in 50 GI polyps using pCLE [3]. Most $(38,76 \%)$ of the polyps were colorectal and gastric polyps were fewer in number $(12,24 \%)$. pCLE was performed after administration of intravenous fluorescein using the standard dosage and technique (as described above). Videos were also recorded for offline evaluation by the same endoscopist at a later date. Considering histopathology as the gold standard, the overall diagnostic accuracy of real-time pCLE and offline examination of the recorded images was $83.3 \%$ and $85.4 \%$, respectively. The diagnostic accuracy in the current study stands in line with the published literature, i.e. $89 \%$ to $94 \%$ in gastric polyps and $72 \%$ to $99 \%$ in colonic polyps. However, a separate analysis was not performed for gastric or colonic polyps. Therefore, it is difficult to ascertain the individual diagnostic accuracy of pCLE in gastric polyps from this study. In addition, the real-time and offline analysis of the images was performed by a single endoscopist. Consequently, the inference regarding inter-observer variability cannot be drawn. Experience of the operator and quality of images for offline evaluation are the other factors that may affect the diagnostic accuracy of pCLE.

Nevertheless, this is the first study from India which evaluated the role of pCLE in GI polypoidal lesions. It is likely that with the improvement in operator's experience, the diagnostic accuracy will increase further.

\section{Conclusions from the study}

The diagnostic performance of pCLE for the in vivo prediction of histology appears reasonably good in the current study. The authors concluded that with the use of CLE, it may be possible to avoid polypectomy in some patients. However, the important question is as follows: how good is good enough? Is the accuracy sufficient to implement the strategy of "resect and discard" or "leave behind" the polyps in the GI tract? In our opinion, the diagnostic accuracy should be at least more than $90 \%$ so that there is a minimum risk of leaving potentially malignant polyps behind. The American Society of Gastrointestinal Endoscopy (ASGE) established the Preservation and Incorporation of Valuable endoscopic
Innovations (PIVI) thresholds for small colorectal polyps. As per the ASGE PIVI threshold criteria, the negative predictive value for adenomatous histology and the agreement of endoscopic technique with histopathology should be $\geq 90 \%$ [31]. Therefore, there is a definite scope of improvement and the use of pCLE for avoiding polypectomy may not be ready for prime time.

\section{CLE: current limitations and future directions}

CLE is a promising imaging technique for various GI lesions. Recent studies have revealed a reasonable degree of accuracy in differentiating neoplastic and non-neoplastic lesions across the GI tract. However, the concerns potentially hampering the widespread use of CLE in clinical practice need to be addressed in future studies. First, most studies depicting excellent results of CLE have been conducted by experts at the academic centers. Consequently, these results may not be reproducible in community practice. For the same reason, there is heterogeneity in the literature in respect to the diagnostic accuracy of these techniques, which may not reach key thresholds for meaningful decisions in community settings. Second, CLE may not be as accurate for small-sized polyps $(<10 \mathrm{~mm})$ as compared to larger polyps [16]. Therefore, the degree of accuracy and negative predictive value of CLE need to be improved especially in cases with smaller polyps. Third, CLE adds to the overall duration and cost of the procedure. Moreover, the field of view is limited as compared to white light endoscopy and NBI. This means that it cannot be used as a sole modality for screening purposes and has to be used in conjunction with wide-field high-definition endoscopy and the other narrow spectrum imaging techniques. Fourth, randomized comparative studies are required between CLE and the currently established narrow spectrum imaging techniques (like NBI and i-scan). Last, the quality of CLE images obtained for subsequent examination or verification is often inadequate. In the published literature, an interpretation of the lesions could not be done in a substantial proportion of patients due to the poor quality of videos obtained during the initial examination $[16,27]$.

In the future, novel devices like dual-axis confocal (DAC) microscopy may overcome some of the drawbacks associated with CLE. In the DAC system, the out-of-focus light is directed away from the pinhole and is more optimally rejected than the single-axis CLE (SAC), thereby improving the signal-tobackground ratio [32]. Preliminary data suggest that DAC may further improve the resolution and the quality of images over the currently available SAC. The development of image interpretation methods and the use of artificial intelligence have gained a great deal of attention over the last few years. It is likely that fully automated computer-aided diagnosis will reduce the dependence on optical diagnosis obtained in real 
time through CLE. In addition, it may reduce the interobserver variability associated with qualitative CLE analysis of images $[33,34]$.

In summary, CLE has the potential to replace conventional histopathology in GI tract lesions provided that quality training, quality assurance, and patient acceptance are addressed adequately. The study in this issue of the Journal is an initial attempt to evaluate the diagnostic accuracy of CLE in Indian patients with gastric and colonic polypoidal lesions. More quality studies are required to establish the role of CLE in clinical practice.

\section{Compliance with ethical standards}

Conflict of interest $\mathrm{ZN}$, and DNR declare that they have no conflict of interest.

Disclaimer The authors are solely responsible for the data and the contents of the paper. In no way, the Honorary Editor-in-Chief, Editorial Board Members, or the printer/publishers are responsible for the results/ findings and content of this article.

\section{References}

1. Longcroft-Wheaton G, Brown J, Cowlishaw D, Higgins B, Bhandari P. High-definition vs standard-definition colonoscopy in the characterization of small colonic polyps: results from a randomized trial. Endoscopy. 2012;44:905-10.

2. Rastogi A, Keighley J, Singh V, et al. High accuracy of narrow band imaging without magnification for the real-time characterization of polyp histology and its comparison with high-definition white light colonoscopy: a prospective study. Am J Gastroenterol. 2009;104: 2422-30.

3. Shah PA, Shah BB, Rai VK, Khan E, Goenka MK. A study on confocal endomicroscopy in comparison with histopathology in polypoidal lesions of the gastrointestinal tract: a prospective single-centre experience. Indian J Gastroenterol. 2019; 38: doi: https://doi.org/10.1007/s12664-019-00973-4.

4. ASGE Technology Committee. Confocal laser endomicroscopy. Gastrointest Endosc. 2014;80:928-38.

5. Kiesslich R, Burg J, Vieth M, et al. Confocal laser endoscopy for diagnosing intraepithelial neoplasias and colorectal cancer in vivo. Gastroenterology. 2004;127:706-13.

6. Xiong YQ, Ma SJ, Zhou JH, Zhong XS, Chen Q. A meta-analysis of confocal laser endomicroscopy for the detection of neoplasia in patients with Barrett's esophagus. J Gastroenterol Hepatol. 2016;31:1102-10.

7. Hurlstone DP, Baraza W, Brown S, Thomson M, Tiffin N, Cross SS. In vivo real-time confocal laser scanning endomicroscopic colonoscopy for the detection and characterization of colorectal neoplasia. Br J Surg. 2008;95:636-45.

8. Gomez V, Buchner AM, Dekker E, et al. Interobserver agreement and accuracy among international experts with probe-based confocal laser endomicroscopy in predicting colorectal neoplasia. Endoscopy. 2010;42:286-91.

9. De Palma GD, Staibano S, Siciliano S, et al. In vivo characterisation of superficial colorectal neoplastic lesions with high-resolution probe-based confocal laser endomicroscopy in combination with video-mosaicing: a feasibility study to enhance routine endoscopy. Dig Liver Dis. 2010;42:791-7.
10. Li WB, Zuo XL, Li CQ, et al. Diagnostic value of confocal laser endomicroscopy for gastric superficial cancerous lesions. Gut. 2011;60:299-306.

11. Jeon SR, Cho WY, Jin SY, Cheon YK, Choi SR, Cho JY. Optical biopsies by confocal endomicroscopy prevent additive endoscopic biopsies before endoscopic submucosal dissection in gastric epithelial neoplasias: a prospective, comparative study. Gastrointest Endosc. 2011;74:772-80.

12. Shahid MW, Buchner AM, Heckman MG, et al. Diagnostic accuracy of probe-based confocal laser endomicroscopy and narrow band imaging for small colorectal polyps: a feasibility study. Am J Gastroenterol. 2012;107:231-9.

13. Kuiper T, van den Broek FJ, van Eeden S, Fockens P, Dekker E. Feasibility and accuracy of confocal endomicroscopy in comparison with narrow-band imaging and chromoendoscopy for the differentiation of colorectal lesions. Am J Gastroenterol. 2012;107: 543-50.

14. Buchner AM, Shahid MW, Heckman MG, et al. Comparison of probe-based confocal laser endomicroscopy with virtual chromoendoscopy for classification of colon polyps. Gastroenterology. 2010;138:834-42.

15. Gong S, Xue HB, Ge ZZ, et al. Value of magnifying endoscopy with narrow-band imaging and confocal laser endomicroscopy in detecting gastric cancerous lesions. Medicine (Baltimore). 2015;94: e1930.

16. Belderbos TDG, van Oijen MGH, Moons LMG, Siersema PD. Implementation of real-time probe-based confocal laser endomicroscopy ( $\mathrm{pCLE}$ ) for differentiation of colorectal polyps during routine colonoscopy. Endosc Int Open. 2017;5:E1104-E10.

17. Chen Q, Cheng HH, Deng S, et al. Diagnosis of superficial gastric lesions together with six gastric lymphoma cases via probe-based confocal laser endomicroscopy: a retrospective observational study. Gastroenterol Res Pract. 2018;2018:5073182.

18. Tontini GE, Mudter J, Vieth M, et al. Prediction of clinical outcomes in Crohn's disease by using confocal laser endomicroscopy: results from a prospective multicenter study. Gastrointest Endosc. 2018;87:1505-14 e3.

19. Karstensen JG, Saftoiu A, Brynskov J, et al. Confocal laser endomicroscopy in ulcerative colitis: a longitudinal study of endomicroscopic changes and response to medical therapy (with videos). Gastrointest Endosc. 2016;84:279-86 e1.

20. Slivka A, Gan I, Jamidar P, et al. Validation of the diagnostic accuracy of probe-based confocal laser endomicroscopy for the characterization of indeterminate biliary strictures: results of a prospective multicenter international study. Gastrointest Endosc. 2015;81:28290.

21. Konda VJ, Meining A, Jamil LH, et al. A pilot study of in vivo identification of pancreatic cystic neoplasms with needle-based confocal laser endomicroscopy under endosonographic guidance. Endoscopy. 2013;45:1006-13.

22. Giovannini M, Caillol F, Monges G, et al. Endoscopic ultrasoundguided needle-based confocal laser endomicroscopy in solid pancreatic masses. Endoscopy. 2016;48:892-8.

23. Gunther U, Daum S, Heller F, et al. Diagnostic value of confocal endomicroscopy in celiac disease. Endoscopy. 2010;42:197-202.

24. Shahid MW, Buchner A, Gomez V, et al. Diagnostic accuracy of probe-based confocal laser endomicroscopy and narrow band imaging in detection of dysplasia in duodenal polyps. J Clin Gastroenterol. 2012;46:382-9.

25. Shahid MW, Buchner AM, Coron E, et al. Diagnostic accuracy of probe-based confocal laser endomicroscopy in detecting residual colorectal neoplasia after EMR: a prospective study. Gastrointest Endosc. 2012;75:525-33.

26. Zhang HP, Yang S, Chen WH, Hu TT, Lin J. The diagnostic value of confocal laser endomicroscopy for gastric cancer and 
precancerous lesions among Asian population: a system review and meta-analysis. Scand J Gastroenterol. 2017;52:382-8.

27. Meining A, Saur D, Bajbouj M, et al. In vivo histopathology for detection of gastrointestinal neoplasia with a portable, confocal miniprobe: an examiner blinded analysis. Clin Gastroenterol Hepatol. 2007;5:1261-7.

28. Buchner AM, Gomez V, Heckman MG, et al. The learning curve of in vivo probe-based confocal laser endomicroscopy for prediction of colorectal neoplasia. Gastrointest Endosc. 2011;73:556-60.

29. Kuiper T, Kiesslich R, Ponsioen C, Fockens P, Dekker E. The learning curve, accuracy, and interobserver agreement of endoscope-based confocal laser endomicroscopy for the differentiation of colorectal lesions. Gastrointest Endosc. 2012;75:1211-7.

30. Yuan XM, Li Z, Ji R, et al. Minimal influence of expertise on the evaluation of colorectal neoplastic lesions by confocal laser endomicroscopy. J Gastroenterol Hepatol. 2014;29:91-5.

31. Rex DK, Kahi C, O'Brien M, et al. The American Society for Gastrointestinal Endoscopy PIVI (Preservation and Incorporation of Valuable endoscopic Innovations) on real-time endoscopic assessment of the histology of diminutive colorectal polyps. Gastrointest Endosc. 2011;73:419-22.

32. Wei L, Yin C, Liu JTC. Dual-axis confocal microscopy for point-ofcare pathology. IEEE J Sel Top Quantum Electron. 2019;25. pii: 7100910.

33. Stefanescu D, Streba C, Cartana ET, Saftoiu A, Gruionu G, Gruionu LG. Computer aided diagnosis for confocal laser endomicroscopy in advanced colorectal adenocarcinoma. PLoS One. 2016;11: e0154863.

34. Queneherve L, David G, Bourreille A, et al. Quantitative assessment of mucosal architecture using computer-based analysis of confocal laser endomicroscopy in inflammatory bowel diseases. Gastrointest Endosc. 2019;89:626-36.

Publisher's note Springer Nature remains neutral with regard to jurisdictional claims in published maps and institutional affiliations. 